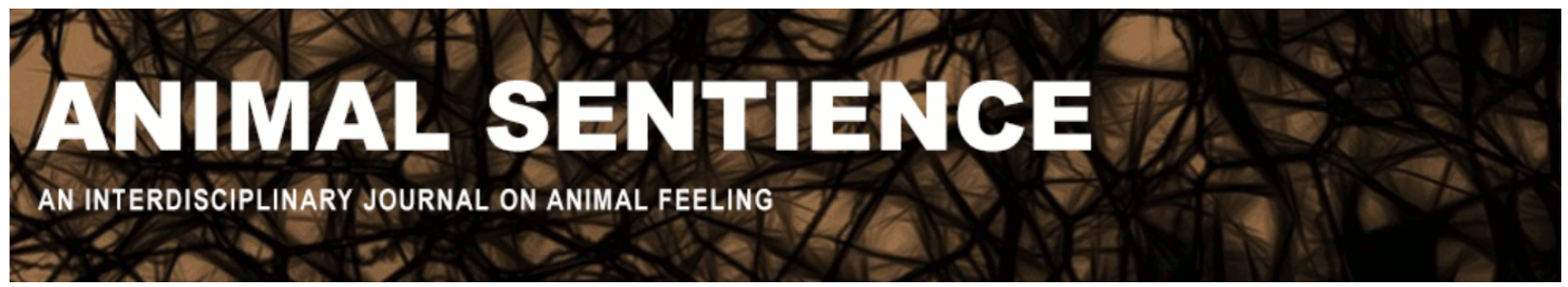

Marino, Lori and Merskin, Debra (2019) Deepening our understanding of sheep. Animal Sentience 25(43)

DOI: $10.51291 / 2377-7478.1510$

Date of submission: 2019-08-09

Date of acceptance: 2019-08-28

(c)

This article has appeared in the journal Animal

Sentience, a peer-reviewed journal on animal

cognition and feeling. It has been made open access,

free for all, by WellBeing International and deposited

in the WBI Studies Repository. For more information,

please contact

wbisr-info@wellbeingintl.org.

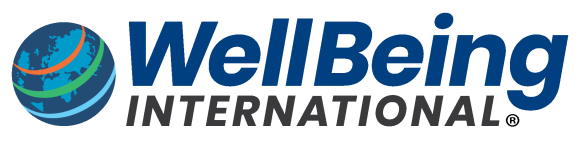

SOLUTIONS FOR PEOPLE, ANIMALS AND ENVIRONMENT 


\title{
Deepening our understanding of sheep
}

Response to Commentary on Marino \& Merskin on Sheep Complexity

\author{
Lori Marino \\ Kimmela Center for Animal Advocacy, Utah \\ Debra Merskin \\ University of Oregon, Eugene
}

\begin{abstract}
Our Response is centered on five major themes: (1) our presentation of human mythologies about sheep; (2) the relevance of cognitive complexity ("intelligence") as a dimension underlying the way people perceive and treat sheep; (3) whether our review is too anthropocentric or anthropomorphic; (4) animal welfare versus animal rights (abolitionism); and (5) whether knowledge and education are enough to change human attitudes and behavior.
\end{abstract}

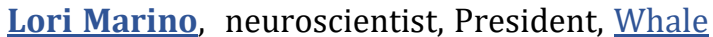
Sanctuary Project, and Executive Director, Kimmela Center for Animal Advocacy, has written over 130 publications on dolphin and whale brain evolution and anatomy; intelligence and self-awareness; and the effects of captivity on social mammals, including cetaceans, elephants and primates. Website

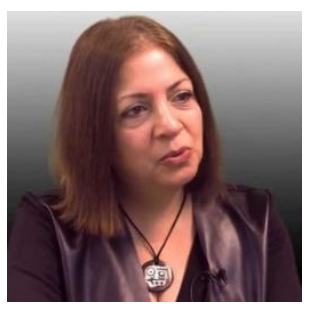

Debra Merskin, Professor of media studies, School of Journalism and Communication, University of Oregon, focuses on how the media and popular press represent animals, resulting in species stereotypes, and how these affect the lived experiences of real animals. Website

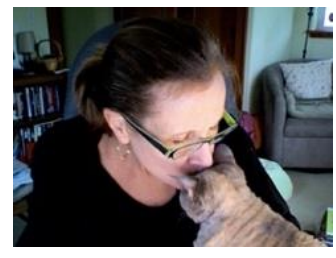

We begin by expressing our appreciation for the many viewpoints offered and for the opportunity to enter into discussion through Open Peer Commentary. The commentaries represent a wide range of thought and opinion on the issues presented in our target article. We would like to begin by reviewing the important elements.

The purpose of our article was to review the scientific literature on sheep cognition, personality, emotions, and sociality. We based our survey on findings from peer-reviewed publications because that is the literature that is the most robust, conservative, and objective among all the different ways one could approach this issue. We did not include anecdotal information because there is no way to confirm it. We chose to focus on findings on the more complex end of the psychological spectrum because we wanted to highlight the ways the psychology of sheep overlaps with that of our own species and other species we consider sophisticated and intelligent. This decision was not due to a lack of concern about 
anthropocentrism. We wanted to position our study and understanding of sheep psychology firmly in basic comparative psychology so the scientific community could better appreciate that sheep are mammals with an evolutionary history and context that goes well beyond their use as commodities.

We concluded that there is abundant evidence that the capacities for learning, cognition, emotion, personality, and social complexity in sheep are on a par with those of many other mammalian (and even primate) species. This contradicts historical and current perceptions of sheep as dull-minded, dependent, and uniform in their nature. We conclude with calls to reconsider the use of sheep as commodities in modern agricultural production and in invasive research, and to study them through noninvasive, non-coercive, and naturalistic methods.

Nearly all the commentators acknowledged the value of reviewing the research evidence on cognition and other psychological attributes in sheep. The views ranged from expressions of confidence that our review would change readers' attitudes towards sheep to reservations that although our review may be a good starting point, it is not enough in itself to change minds. Most views fell in between. The five themes that underlie most of the points made in the commentaries concerned: (1) the completeness and accuracy of our presentation of human mythologies about sheep; (2) the relevance of cognitive complexity ("intelligence") to how we perceive and treat sheep; (3) anthropocentrism and anthropomorphism; (4) welfare versus rights (abolitionism); and (5) whether knowledge and education are enough to change human minds, hearts and behavior.

\section{Sheep Mythology}

In popular usage, "mythology" refers to narratives that may be fictional rather than factual. Depth psychology, however, teaches us that myths are among the most important stories cultures tell about themselves, answering life's big questions. Animals are often used as symbolic stand-ins for humans, experiencing trials and tribulations; they make it easier to explain (and teach to children) about origins, love, death, and ethics. We are now "modern," but this does not mean the view of nonhuman animals we have learned from fables and fairytales is gone. These stories were created at a time when humans lived in much closer relationships with other animals and human knowledge of animals was transmitted in both literal and symbolic ways (Merskin 2018). Today, however, for some of us, the tales we tell about other animals (whether in stories, film, or advertisements) are the only contact we have with them. In our article, we explored potential reasons sheep and lambs might be misunderstood, their individual natures minimized, and their behaviors stereotyped.

Several commentators (Franklin; Porcher; Hermanson; Colombo \& Raucea) focus on this introductory aspect of our target article. Colombo \& Raucea feel we have misunderstood literary and poetic portrayals of sheep. Hermanson finds the literary and cultural history less relevant than economics to understanding human perception and treatment of animals. Vonk, Horback, and Gygax \& Nawroth criticize our article as anthropomorphic. Sevillano makes the connection between popular cultural portrayals and sheep stereotypes. Franklin goes further, referring to sheep as "baby-faced" in a neotenized reading of sheep facial features. We don't necessarily agree. Many do have large eyes and ears, especially lambs, but they do not evoke "babyish cues" as strongly as other animals do. 
Stereotype theory certainly does produce one-dimensional assessments of others on the basis of some perceived difference, as Franklin notes.

The commentaries include some terrific images illustrating the ways many people who never interact with real sheep are likely to have cultivated stereotypical views (as in other -isms such as racism and sexism); this supports our suggestion that children often learn about sheep and other animals from cartoons and stories. Some commend our inclusion of sheep mythology but argue that what we present is inaccurate. Porcher, for example, agrees that there is "a blind spot in science" and that The Great Chain of Being and folkloric understanding can reveal the source of stereotypes and prejudices about sheep. She develops our argument that stories and tales about sheep construct cultural views that support their erasure as individuals and plants them firmly in the role of use-object: "Biology's investment in agribusiness, the pharmaceutical industry, the pesticide industry, the biological warfare industry, the fishing industry, and the leather and fur industries, to name just a few, shows the same pattern of treating nature and animals as being there to serve the human project only."

Colombo \& Raucea argue that in fables, sheep are not docile. Their review of the literature on fables and stories suggests that sheep are not portrayed as dumb or passive. This may be true, but there are also ample examples where sheep are presented as less adventuresome or aggressive than other species, and those portrayals are based on beliefs that they are devoid of personality and intelligence. Our focus was not specifically on popular portrayals of sheep; art, advertising, myth, and tradition paint particular pictures of them. What Colombo \& Raucea discuss could be an essay in itself. We agree that sheep, like most other victims of torture and slaughter, are not passive recipients of such treatment. We were not trying to perpetuate stereotypes of sheep but to expose how they serve as justification for mistreatment and harm and how they are contradicted by research.

Hermanson agrees about the need for greater protection but questions whether mythological portrayals have as much influence as we might think. He suggests that economics is a more important causal factor: "The problem is not discourses of production; it is production itself. Indeed, the 19th amendment did not depend on discovering that women could recognize faces or exploding the stereotype that women are one-dimensional non-individuals. Female, and male, disenfranchisement had more to do with property" (in other words, their being property). Hermanson also reminds us that racism and sexism are intimately connected with speciesism and that economics is what sustains inequitable treatment in all three cases. Economics means power. We continue to suggest, however, that belief systems grounded in mythology help to "normalize" hegemonic attitudes.

\section{Relevance of "Intelligence"}

Several commentators (Browning, Palmer \& Sandøe; Woodford \& Carter, and Vonk) ask which is more relevant to how we should treat sheep: their cognitive capacity or their capacity to suffer? This is a critical issue in animal ethics. Our aim was to review the full scope of sheep psychology, not just the psychological dimensions most relevant to suffering; but we agree that the ability to suffer is particularly important. Cognitive and social complexity are themselves relevant to suffering, however, shaping the adaptation and even the awareness of different species to various circumstances. Hence the relation between cognitive capacity and suffering is worth analyzing. 
Many commentators (Colombo \& Scarf; Rollin; Phillips; D'Silva; King; Paez; Kendrick) recognize the importance of increasing our understanding of sheep psychology "across the board." Li emphasizes recognizing the complexity and individuality of sheep and other animals for a better perspective on our own species' claims of superiority. A number of commentators suggest that intelligence and cognitive complexity come into play in more practical terms in studying how sheep learn to cope in various situations (Webster) and how enriching their environment and devices can enhance their well-being (Browning; Found). Found emphasizes the potential contribution of research findings on domestic and wild ungulates to welfare policies and conservation strategies. Yet knowledge itself is not sufficient to change perspectives and behavior (as discussed in section 5, below). Hanlon voices frustration that we still need to "prove" that other animals are complex, sentient creatures, capable of suffering, in order to justify protecting them (even inadequately). We share her sentiments.

McLennan points out that research findings should be applied to providing appropriate treatment for sheep and other animals. We agree that welfare practices must be based on data. Kiley-Worthington, on the other hand, rejects a scientific and objective approach to studying sheep and other animals in favor of a more subjective, anecdotal approach. She is dismissive of the methodology we used and suggests that shepherds and others who interact with sheep daily already know that sheep are capable of the capacities we discuss. The problem with "folk psychology" is that it does not protect against bias: shepherds and others who "work with the animals" on a daily basis in a context of exploiting them are going to view the animals in an instrumental way, shaped by their use.

\section{Anthropocentrism/Anthropomorphism}

Our target article was criticized for being too anthropocentric by some commentators and for being too anthropomorphic by others. Anthropocentrism measures other animals' capacities in terms of our own, not taking into account species differences in evolutionary-adaptive history. Anthropocentrism places humans at the top of a scale in which no other animal can measure up (Chapman \& Huffman 2018). Adolphs, Brooks Pribac, Figdor, Davis, PeñaGuzman, and Pellis all make this important point. They argue that sheep and other animals should be studied and understood on their own terms. Although we focused selectively on refereed research findings, we agree completely that much of this literature is based on a human-centered view of intelligence and that it underestimates the natural abilities of other animals. This general problem with how we study and draw conclusions about other animals in comparative psychology goes beyond the scope of our target article. The predilection of our own species for using ourselves as a measuring stick is a cognitive limitation, as argued forcefully by Davis.

We wish to thank Brodbeck, Brodbeck \& Rosso for making the point that domesticated animals, like sheep, have been selected (artificially, by humans) for temperamental traits such as shyness. Shy humans can be misperceived as being less intelligent; we should not make the same mistake (anthropomorphizing) about sheep and other animals. Brodbeck et al. provide a nice summary of many of the traits we reviewed in sheep and of their occurrence in other animals. Brennan adds that even wild, nondomesticated ungulates are subject to biased views about their intelligence and complexity. 
On the other end of the spectrum, Vonk, Horback, Correia Caeiro, and Gygax \& Nawroth do not find our review as objective as they would hope, suggesting that we draw exaggerated conclusions about the intelligence of sheep. Vonk suggests that we have "cherrypicked" the evidence. Mallatt finds this criticism too strong, given that "most of the evidence validly supports the claims," but he is concerned about the lack of "negative" evidence. We agree that anthropomorphism - the attribution of human traits to nonhumans who do not possess those traits - is to be avoided. But anthropomorphism is only anthropomorphism when the "human" trait does not actually exist in the other animal. What we were doing in our review was highlighting the evidence of psychological capacities that sheep share with other species. Reviewing "negative" evidence is difficult when the objective is to review the positive evidence: there no doubt exists also a whole range of abilities for which there is no evidence in sheep.

Gamez, like Vonk, makes the point that we seem to over-interpret the findings on sheep intelligence and that many aspects of sheep psychology could be "automatic." Many cognitive capacities in all species, including humans, are indeed unconscious or over-learned. Gamez provides the example of personality and the dimension of shyness and boldness. His example of emotional responses contingent on spatial distance from other sheep does not make sense in terms of what we know personality to be: a consistent tendency to behave in a certain way across time under the same circumstances. Gamez's hypothesis about facial perception in sheep being explainable by simple input-output mechanisms is not evidence that this is indeed how sheep process faces or even that there is sufficient data to suggest this as a reasonable psychological explanation. We do agree with Gamez, however, that flexible hypothesis-testing abilities are hallmarks of complex intelligence and that there is evidence for these abilities in sheep.

Abbate's criticism is similar to that of Kiley-Worthington in that she promotes the use of anecdotes in a systematic review of intelligence and cognition in sheep (or any nonhuman animal). She suggests that "important anecdotes" are missing from our article. We explicitly set out to keep anecdotal information and hearsay out of our review for the very reason we explained above in responding to Kiley-Worthington. Anecdotal evidence (especially popular accounts of animal behavior) is unreliable. It is not clear whether Abbate is suggesting that the mirror self-recognition test is too narrowly focused or that it is overinterpreted by some authors. Nor is it clear that it is anthropocentric.

Willett, too, seems to misunderstand the mirror self-recognition literature. She thinks other sensory modalities such as smell may provide better ways to probe self-awareness, suggesting that the "yellow snow" studies with dogs demonstrate self-awareness in the olfactory realm. Self-awareness is not modality-specific; but an experiment on whether dogs can discriminate their own urine from that of others is not a test of self-awareness. It is simply a test of discrimination. Willett suggests that sheep may not "pass" the mirror test because that is not where their "emotive center" lies. We agree that motivation is important, but Willett may be reading too much into the logic of the self-recognition protocol.

\section{Welfare Versus Rights}

That more knowledge about what is going on in the mind of a sheep (or any animal) ought to promote better treatment is shared by many commentators but there were differences as to what that "better treatment" should be. This leads to the debate between "welfarism" (make 
animal use less harmful) versus animal rights and "abolitionism" (put an end to all animal use), which was articulated in more detail in Marino (2016). It is encouraging to see that the majority of our peers recognize the importance of promoting well-being in sheep but we find ourselves in the minority in our stance regarding the sufficiency of welfare-based policies alone. Improving animal welfare is critically important; this is especially well-articulated by D'Silva, Woodruff, Horback, and Mehrkam. But welfarism still gives priority to human interests over the needs of other animals. Hence welfarism does not "move the needle" in our relationship with sheep and other animals. Despite decades of welfare efforts, other animals, including farmed sheep, are mostly worse off than ever before. The number of sheep killed for food is growing and sheep are subjected to increasingly invasive and harmful biomedical procedures.

We think welfare-based policies alone are not enough to remedy suffering in farmed animals. We do not wish simply to create better conditions for exploitation: we would like to see animal use and exploitation phased out entirely. As Li has pointed out, welfarism does not call into question our assumptions about how humans should relate to other animals. (D'Silva comes the closest). Welfarism eschews the issue of animal rights entirely. Woodford \& Carter claim that an abolitionist stance towards sheep is "problematic" because domestic sheep are creations of artificial breeding, hence it does not make sense to "re-wild" them. But nowhere do we suggest that domesticated sheep should be "re-wilded." We are simply pointing to the evidence that domesticated sheep have feelings, and lives to live, and that they should be viewed and treated as such. Our target article did not ask which practices and which levels of suffering are somehow permissible. Our aim was to lay out the empirical evidence about sheep intelligence, emotion, personality, and sociality, not to enter into abstract ethical debate. If it is not self-evident that neither sheep, nor any sentient being, should be treated the way they are being treated by our species, we are at a loss as to how any ethical theorizing would resolve the issue. Only Paez explicitly promotes the notion that we must not only provide better welfare for sheep: we should not harm them. Taken seriously, this is an argument for abolition, because despite the best intentions of welfare practitioners, harm is inherent in the use of other animals as a means to our ends.

\section{Is Knowledge Enough?}

The most important question raised by the peer commentaries is whether simply educating people about the facts is only necessary but not sufficient for changing attitudes toward the treatment of other animals. The work of The Someone Project, to which our target article belongs, is based on the hypothesis that for people to understand "who" is on their plate, they need to be supplied with the empirical facts about the psychology of farmed animals. Phillips and Rollin are among the commentators who think that information alone should be enough. Other commentators, however, point out that education has often proved insufficient to change people's views of other animals. Mallatt and Gradidge \& Zawisza make this point especially well. We agree that it is critically important to examine the circumstances under which information succeeds and fails to motivate attitude change. Our target article was intended as a first step in deepening human understanding and respect for sheep. We view this step as necessary but not enough. Understanding individual differences in what is needed to change behavior will be essential in this kind of research, and in advocacy of any kind. 


\section{Conclusion}

We thank all our peers who submitted commentaries on the target article. Our own understanding has been enhanced by their comments and the challenge of responding. We hope the target article, the 41 thoughtful commentaries, and this Response may prompt readers to think more deeply about their perception and treatment of sheep and other animals.

\section{References}

Abbate, C. (2019). Sheep complexity outside the laboratory. Animal Sentience 25(21). Adolphs, R. (2019). Using sheep psychology to guide sheep policy. Animal Sentience 25(14). Brennan, 0. (2019). Complexity of wild ruminants. Animal Sentience 25(38).

Brodbeck, D. R., Brodbeck, M. I. R., \& Rosso, K. (2019). Domestication and cognitive complexity. Animal Sentience 25(32).

Brooks-Pribac, T. (2019). Positive sentience is underrated. Animal Sentience 25(11).

Browning, H. (2019). What should we do about sheep? The role of intelligence in welfare considerations. Animal Sentience 25(23).

Chapman, C. A., \& Huffman, M. A. (2018). Why do we want to think humans are different? Animal Sentience 23(1).

Colombo, M., \& Raucea, C. (2019). Sheep in Aesop's and Phaedrus's fables. Animal Sentience 25(13).

Colombo, M., \& Scarf, D. (2019). Sheeple? The need for more research on sheep cognition. Animal Sentience 25(12).

Correia Caeiro, C. (2019). Cognitive dissonance about sheep cognition and consumption. Animal Sentience 25(39).

D'Silva, J. (2019). Reflections on sheep rearing. Animal Sentience 25(5).

Davis, H. (2019). Our disparaging view of sheep is indeed based on cognitive inadequacy: Unfortunately, it's ours. Animal Sentience 25(20).

Figdor, C. (2019). The mental lives of sheep and the quest for a psychological taxonomy. Animal Sentience 25(16).

Found, R. (2019). Cognition, emotion, personality, and the conservation of wild ungulates. Animal Sentience 25(22).

Franklin, R. G., Jr. (2019). Why are sheep sheepish? How perception affects animal stereotyping. Animal Sentience 25(15).

Gamez, D. (2019). The intelligence of sheep. Animal Sentience 25(27).

Gradidge, S., \& Zawisza, M. (2019). Why factual appeals about the abilities of sheep may fail. Animal Sentience 25(42).

Gygax, L., \& Nawroth, C. (2019). Farm animals are not humans in sheep clothing. Animal Sentience 25(25).

Hanlon, A. (2019). Sheep are sentient, but not identical. Animal Sentience 25(41).

Hermanson, S. (2019). The problem is not discourses of production, it is production itself. Animal Sentience 25(29).

Horback, K. (2019). Applied cognition research to improve sheep welfare. Animal Sentience 25(18). 
Kendrick, K. M. (2019). Social cognition in sheep: Welfare implications. Animal Sentience 25(40).

Kiley-Worthington, M. (2019). Anthropomorphism is the first step. Animal Sentience 25(30). King, J. (2019). Adding sheep to the spectrum of comparative psychology. Animal Sentience 25(33).

Li, P. J. (2019). Debunking human prejudice and blindness. Animal Sentience 25(17).

Mallatt, J. (2019). Time to vary the formula. Animal Sentience 25(28).

Marino, L. (2016). Why animal welfarism continues to fail. Animal Sentience 7(5).

McLennan, K. (2019). The importance of research applicability. Animal Sentience 25(31).

Mehrkam, L. R. (2019). A behaviorist approach to sheep cognition, intelligence, and welfare. Animal Sentience 25(37).

Merskin, D. (2018). Seeing species: Re-presentations of animals in media and popular culture. New York: Peter Lang.

Paez, E. (2019). Becoming the good shepherds. Animal Sentience 25(30).

Palmer, C., \& Sandøe, P. (2019). Yes, sheep are smart but the moral question is still 'can they suffer'? Animal Sentience 25(24).

Pellis, S. M. (2019). Guilty as charged. Animal Sentience 25(19).

Peña-Guzman, D. M. (2019). Casting a sheep's eye on science. Animal Sentience 25(6).

Phillips, C. (2019). Sacrificial lambs. Animal Sentience 25(2).

Porcher, I. F. (2019). On the sentience of sheep and other useful objects. Animal Sentience 25(8).

Rollin, B. (2019). Far more to sheep than meets the casual eye. Animal Sentience 25(7).

Sevillano, V. (2019). Our ambivalent stereotypes of sheep. Animal Sentience 25(35).

Vonk, J. (2019). Pulling the wool from our eyes. Animal Sentience 25(3).

Webster, J. (2019). Sentient animals do not just live in the present. Animal Sentience 25(10).

Willett, C. (2019). Can a mirror capture the self? Animal Sentience 25(36).

Woodford, P., \& Carter, A. (2019). Science, social critique, and the need for ethics. Animal Sentience 25(34).

Woodruff, M. L. (2019). Smart sheep need more protection. Animal Sentience 25(9). 